\title{
Hall Effects on Unsteady MHD Flow Between Two Rotating Disks with Non-Coincident Parallel Axes Embedded in a Porous Medium
}

\author{
S. Das \\ Department of Mathematics \\ University of Gour Banga \\ Malda 732 103, India
}

\author{
B.C. Sarkar \\ Department of \\ Applied Mathematics \\ Vidyasagar University \\ Midnapore 721 102, India
}

\author{
R. N. Jana \\ Department of \\ Applied Mathematics \\ Vidyasagar University \\ Midnapore 721 102, India
}

\begin{abstract}
Hall effects on an unsteady MHD flow of a viscous incompressible electrically conducting fluid between two rotating disks with non-coincident parallel axes embedded in a porous medium have been studied. The governing equations have been solved analytically using the Laplace transform technique. The effects of rotation parameter, Hall parameter, Hartmann number and Darcy number have been considered on the flow characteristics and illustrated by graphs. It is observed that the velocity components are significantly affected by Hall parameter and Darcy number. The velocity components increase near the lower disk whereas they decrease near the upper disk with an increase in either rotation parameter of disk or rotation parameter of axis. Further, the torque experienced by the disks increases with an increase in either Hartmann number or rotation parameter while it decreases with an increase in either Hall parameter or Darcy number.
\end{abstract}

Keywords: Hall effects, Hartmann number, rotation parameters, rotating disks, non-coincident, porous medium.

\section{INTRODUCTION}

The rotating flows over rotating disks or bodies through a porous medium have numerous applications in meteorology, geophysical and cosmical fluid dynamics. It is also important in the areas such as computer storage devices, viscometry and rotating machinery, aerospace, extraction process of fluid from the porous ground, lubrication of porous bearings, chemical engineering as well as biophysics, electrochemistry and materials processing. The hydrodynamic flow between two disks, rotating about the same axis, has been studied by Stewartson [1], Rott and Lewellen [2], Mellor et al.[3], Erdogan[4], Ersoy [5] and many others. The hydrodynamic flow between two non-coincident rotating disks embedded in a porous media has been discussed by Jana et al.[6]. The study of a magnetohydrodynamic (MHD) flow between two rotating disks plays a dignified representation of rotating flows with the axisymmetric solution of the problem. Mohanty [7] has studied the hydromagnetic flow between two rotating disks with noncoincident parallel axes of rotation. Guria et al. [8] have studied the unsteady MHD flow between two disks with non-coincident parallel axes of rotation. The hydromagnetic flow between two porous disks rotating about non-coincident axes has been discussed by Guria et al.[9].

The Hall effects are important when the magnetic field is high or when the collision frequency is low (Sutton and Sherman [10]). The current induced in a fluid is usually carried predominantly by electrons, which are considerably more mobile than ions. The electron drift velocity in most cases leads to a second component of the flow velocity, which in turn leads to a secondary force and causes anisotropic electrical conductivity in the flow. The current component created by this anisotropic conductivity is known as the Hall current. The dimensionless product $\omega_{e} \tau_{e}$, usually called the Hall parameter, is an important characteristic number in the MHD design, where $\omega_{e}$ is the electron cyclotron frequency and $\tau_{e}$ the electron collision mean free time. On the microscopic scale, the Hall parameter indicates the average angular travel of electrons between collisions, while on the macroscopic scale, the value of $\omega_{e} \tau_{e}$ indicates the relative importance of the Hall field and the Hall current. If both the electron $\left(\omega_{e} \tau_{e}\right)$ and the ion $\left(\omega_{i} \tau_{i}\right)$ Hall parameters are large simultaneously as the case in some weakly ionized gases, the conductivity is then reduced due to a phenomenon called ion-slip. Though $\tau_{i}$ is ordinarily larger than $\tau_{e}, \omega_{i}$ is much smaller than $\omega_{e}$. Therefore, the product $\omega_{i} \tau_{i}$ is usually negligible (Tillack and Morley [11]). It is obvious from the above analysis that the Hall effects cannot be neglected when the electron cyclotron frequency is relatively high. The problems related to these Hall currents have important engineering applications in MHD power generation and of Hall accelerators as well as in flight magnetohydrodynamics. Taking this fact into account, the effects of Hall currents on the hydromagnetic flow under different geometries have been studied by Sato [12], Yamanishi [13], Sherman and Sutton [14], Gupta [15], Datta and Jana [16, 17], Jana and Datta [18] etc. Kanch and Jana [19] have investigated the Hall effects on the hydromagnetic flow between two rotating disks with noncoincident parallel axes of rotation. The Hall effects on an unsteady MHD flow between two disks with non-coincident parallel axes of rotation have been investigated by Das et al.[20]. Recently, Barik et al. [21] have studied the Hall effects on an unsteady MHD flow between two rotating disks with non-coincident parallel axes.

The present paper is devoted to study the effects of Hall currents as well as rotating axis about which the disk rotates through a porous medium. The axes are also rotating with an uniform angular velocity $\Omega$. The governing equations have been solved analytically using the Laplace transform technique. The effects of various parameters of the problem are discussed. In the light of our present analysis it is stated that there arises symmetric motion about the mid plane between the two disks as referred to the rigid body rotations.

\section{MATHEMATICAL FORMULATION AND ITS SOLUTION}

Consider the unsteady flow of a viscous incompressible electrically conducting fluid through a porous medium between two parallel disks rotating with same uniform angular velocity $\omega$ about two different axes at a distance $a$ apart. The axes are also rotating with an uniform angular velocity $\Omega$. We choose a 
cylindrical polar co-ordinates system $(r, \theta, z)$ with the axis normal to the disks situated symmetrically between two axes of rotation (see Fig.1). The two axes of rotation of the disks are parallel to the $z$-axis. A uniform transverse magnetic field $B_{0}$ is applied perpendicular to the disks.

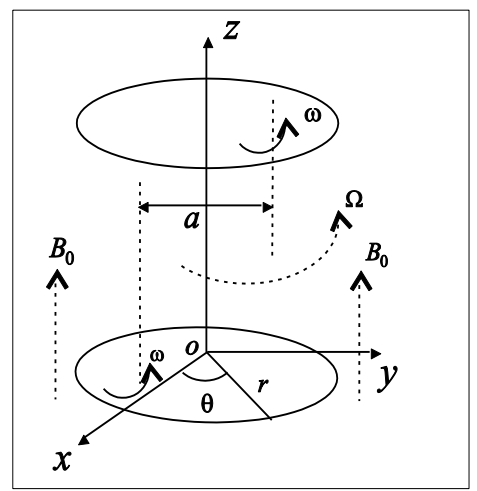

Fig.1: Geometry of the problem

The Navier-Stokes equations along $r, \theta$ and $z$-directions are

$$
\begin{aligned}
\frac{\partial u}{\partial t}+ & u \frac{\partial u}{\partial r}+\frac{v}{r} \frac{\partial u}{\partial \theta}+w \frac{\partial u}{\partial z}-\frac{v^{2}}{r}-2 \Omega v \\
= & -\frac{1}{\rho} \frac{\partial p}{\partial r}+v\left[\frac{\partial^{2} u}{\partial r^{2}}+\frac{1}{r} \frac{\partial u}{\partial r}+\frac{1}{r^{2}} \frac{\partial^{2} u}{\partial \theta^{2}}+\frac{\partial^{2} u}{\partial z^{2}}-\frac{u}{r^{2}}\right. \\
& \left.-\frac{2}{r^{2}} \frac{\partial v}{\partial \theta}-\frac{u}{k^{*}}\right]+\frac{B_{0}}{\rho} j_{\theta}, \\
\frac{\partial v}{\partial t}+ & u \frac{\partial v}{\partial r}+\frac{v}{r} \frac{\partial v}{\partial \theta}+w \frac{\partial v}{\partial z}+\frac{u v}{r}+2 \Omega u \\
= & -\frac{1}{\rho r} \frac{\partial p}{\partial \theta}+v\left[\frac{\partial^{2} v}{\partial r^{2}}+\frac{1}{r} \frac{\partial v}{\partial r}+\frac{1}{r^{2}} \frac{\partial^{2} v}{\partial \theta^{2}}+\frac{\partial^{2} v}{\partial z^{2}}-\frac{v}{r^{2}}\right. \\
& \left.+\frac{2}{r^{2}} \frac{\partial u}{\partial \theta}-\frac{v}{k^{*}}\right]-\frac{B_{0}}{\rho} j_{r}, \\
\frac{\partial w}{\partial t}+ & u \frac{\partial w}{\partial r}+\frac{v}{r} \frac{\partial w}{\partial \theta}+w \frac{\partial w}{\partial z} \\
= & -\frac{1}{\rho} \frac{\partial p}{\partial z}+v\left[\frac{\partial^{2} w}{\partial r^{2}}+\frac{1}{r} \frac{\partial w}{\partial r}+\frac{1}{r^{2}} \frac{\partial^{2} w}{\partial \theta^{2}}+\frac{\partial^{2} w}{\partial z^{2}}-\frac{w}{k^{*}}\right],
\end{aligned}
$$

where $\vec{q} \equiv(u, v, w), \vec{B} \equiv\left(B_{r}, B_{\theta}, B_{0}\right), \vec{j} \equiv\left(j_{r}, j_{\theta}, j_{z}\right), \quad p, \rho$ $k^{*}$ and $v$ are respectively the velocity vector, the magnetic field vector, the current density vector, the fluid pressure, the density of the fluid, the permeability of the porous medium and the kinematic viscosity.

We assume that the magnetic Reynolds number is small so that the induced magnetic field is neglected. This assumption is reasonable for the flow of liquid metals, e.g. mercury or liquid sodium which are electrically conducting under laboratory conditions. The electron-atom collision frequency is assumed to be relatively high so that the Hall effects can be included. The generalized Ohm's law on taking Hall currents into account is (see Cowling [22])

$$
\vec{j}+\frac{\omega_{e} \tau_{e}}{B_{0}}(\vec{j} \times \vec{B})=\sigma(\vec{E}+\vec{q} \times \vec{B}),
$$

where $\vec{E} \equiv\left(E_{r}, E_{\theta}, E_{z}\right)$ is the electric field vector, $\omega_{e}$ the cyclotron frequency, $\tau_{e}$ the electron collision time and $\sigma$ the electrical conductivity of the fluid. Maxwell's equations are

$$
\nabla \times \vec{B}=\mu_{e} \vec{j}, \nabla \times \vec{E}=-\frac{\partial \vec{B}}{\partial t}, \nabla \cdot \vec{B}=0, \nabla \cdot \vec{D}=\rho_{e},
$$

where $\mu_{e}$ is the magnetic permeability, $\vec{D}$ the displacement current vector and $\rho_{e}$ the free charge density.

The equation of the conservation of current is

$$
\nabla \cdot \vec{j}=0 \text {. }
$$

The equation of continuity $\nabla \cdot \vec{q}=0$ with no-slip condition at the disks gives $w=0$ everywhere in the flow. The solenoidal relation $\nabla \cdot \vec{B}=0$ gives $B_{z}=$ constant $=B_{0}$ everywhere in the flow.

In view of the above assumptions, the equation (4) yields

$$
\begin{aligned}
& j_{r}+m j_{\theta}=\sigma\left(E_{r}+B_{0} v\right), \\
& j_{\theta}-m j_{r}=\sigma\left(E_{\theta}-B_{0} u\right), \\
& j_{z}=\sigma E_{z},
\end{aligned}
$$

where $m=\omega_{e} \tau_{e}$ is the Hall parameter.

Solving for $j_{r}$ and $j_{\theta}$, we have

$$
\begin{aligned}
& j_{r}=\frac{\sigma}{1+m^{2}}\left[\left(E_{r}+B_{0} v\right)-m\left(E_{\theta}-B_{0} u\right)\right], \\
& j_{\theta}=\frac{\sigma}{1+m^{2}}\left[m\left(E_{r}+B_{0} v\right)+\left(E_{\theta}-B_{0} u\right)\right] .
\end{aligned}
$$

The initial and boundary conditions are

$$
\begin{aligned}
& u(z, 0)=0, v(z, 0)=0, w=0 \text { for all } 0 \leq z \leq h \\
& u=-\frac{1}{2} \omega a \cos \theta, v=\omega\left(r+\frac{1}{2} a \sin \theta\right), w=0 \text { at } z=0 \text { for } t>0, \\
& u=\frac{1}{2} \omega a \cos \theta, v=\omega\left(r-\frac{1}{2} a \sin \theta\right), w=0 \text { at } z=h \text { for } t>0 .
\end{aligned}
$$

The equation (6) gives $j_{z}=$ constant . This constant is zero since $j_{z}=0$ at the disks which are electrically non-conducting. Thus $j_{z}=0$ everywhere in the flow. Hence $j_{z}=\sigma E_{z}=0$ in the fluid everywhere.

On the use of the equation (5), we get

$$
\frac{\partial E_{\theta}}{\partial z}=0, \frac{\partial E_{r}}{\partial z}=0, \frac{\partial}{\partial r}\left(r E_{\theta}\right)-\frac{\partial E_{r}}{\partial \theta}=0 .
$$

The equation (13) shows that the electric field is independent of $z$.

In respect of the initial and boundary conditions (12), we assume the velocity and pressure distributions as

$$
\begin{aligned}
& u=A(z, t) \cos \theta+B(z, t) \sin \theta, \\
& v=\omega r+B(z, t) \cos \theta-A(z, t) \sin \theta, \\
& w=0 \text { and } p=p_{0}+\frac{1}{2} \rho \Omega^{2} r^{2}+\rho \Omega \omega r^{2}+\rho g^{*} z,
\end{aligned}
$$

where $p_{0}$ is a constant and $g^{*}$ the gravitational acceleration. Integrating $j_{r}$ and $j_{\theta}$ between the limits $z=0$ and $z=h$ and putting

$$
\int_{0}^{h} j_{r} d z=0 \text { and } \int_{0}^{h} j_{\theta} d z=0
$$

we get, on using (10) and (11)

$$
\begin{aligned}
& E_{r}=-\frac{B_{0}}{h}(\omega r h+P \cos \theta-Q \sin \theta), \\
& E_{\theta}=\frac{B_{0}}{h}(Q \cos \theta+P \sin \theta),
\end{aligned}
$$

where

$$
P=\int_{0}^{h} B(z, t) d z \text { and } Q=\int_{0}^{h} A(z, t) d z .
$$

It is noticed that the expressions given by (16) for $E_{r}$ and $E_{\theta}$ 
are satisfied with equations (13).

It can be easily proved that $A(z, t)$ and $B(z, t)$ satisfy the following equations

$$
\begin{aligned}
& \frac{\partial A}{\partial t}-(\omega+2 \Omega) B \\
& =v \frac{\partial^{2} A}{\partial z^{2}}+\frac{\sigma B_{0}^{2}}{\rho\left(1+m^{2}\right)}\left[\left(\frac{Q}{h}-A\right)-m\left(\frac{P}{h}-B\right)\right]-\frac{v}{k^{*}} A, \\
& \frac{\partial B}{\partial t}+(\omega+2 \Omega) A \\
& \quad=v \frac{\partial^{2} B}{\partial z^{2}}+\frac{\sigma B_{0}^{2}}{\rho\left(1+m^{2}\right)}\left[m\left(\frac{Q}{h}-A\right)+\left(\frac{P}{h}-B\right)\right]-\frac{v}{k^{*}} B .
\end{aligned}
$$

On the use of (14), the initial and boundary conditions (12) becomes

$$
\begin{aligned}
& A(z, 0)=0, B(z, 0)=0 \text { for } 0 \leq z \leq h, \\
& A(0, t)=-\frac{1}{2} \omega a, B(0, t)=0 \text { for } t>0, \\
& A(h, t)=\frac{1}{2} \omega a, B(h, t)=0 \text { for } t>0 .
\end{aligned}
$$

Introducing the non-dimensional variables

$$
f=\frac{A}{a \omega}, g=\frac{B}{a \omega}, \eta=\frac{z}{h}, \tau=\frac{v t}{h^{2}}, c=\frac{Q}{\omega a h}, d=\frac{P}{a \omega h},
$$

equations (18) and (19) can be written as

$$
\begin{aligned}
\frac{\partial f}{\partial \tau} & -\left(K^{2}+2 K_{1}^{2}\right) g \\
= & \frac{\partial^{2} f}{\partial \eta^{2}}+\frac{M^{2}}{1+m^{2}}[(c-f)-m(d-g)]-\frac{1}{D a} f, \\
\frac{\partial g}{\partial \tau}+ & \left(K^{2}+2 K_{1}^{2}\right) f \\
= & \frac{\partial^{2} g}{\partial \eta^{2}}+\frac{M^{2}}{1+m^{2}}[m(c-f)+(d-g)]-\frac{1}{D a} g,
\end{aligned}
$$

where $M=\left(\frac{\sigma}{\rho v}\right)^{\frac{1}{2}} B_{0} h$ is the Hartmann number, $K^{2}=\frac{\omega h^{2}}{v}$ the rotation parameter of the disk, $K_{1}^{2}=\frac{\Omega h^{2}}{v}$ the rotation parameter of the axis and $D a=\frac{k^{*}}{h^{2}}$ the Darcy number.

The initial and boundary conditions (20) become

$$
\begin{aligned}
& f(\eta, 0)=0, g(\eta, 0)=0 \text { for } 0 \leq \eta \leq 1, \\
& f(0, \tau)=-\frac{1}{2}, g(0, \tau)=0 \text { for } \tau>0, \\
& f(1, \tau)=\frac{1}{2}, g(1, \tau)=0 \text { for } \tau>0 .
\end{aligned}
$$

Combining equations (22) and (23), we get

$$
\begin{aligned}
& \frac{\partial^{2} F}{\partial \eta^{2}}-\left[\frac{M^{2}}{1+m^{2}}+\frac{1}{D a}+i\left(\frac{m M^{2}}{1+m^{2}}+K^{2}+2 K_{1}^{2}\right)\right] F \\
& =\frac{\partial F}{\partial \tau}-\frac{M^{2}(1+i m)}{1+m^{2}} n^{*}
\end{aligned}
$$

where

$$
F=f+i g \text { and } n^{*}=c+i d=\int_{0}^{1} F(\eta, \tau) d \eta .
$$

The initial and boundary conditions (24) reduce to

$$
\begin{aligned}
& F(\eta, 0)=0 \text { for all } 0 \leq \eta \leq 1, \\
& F(0, \tau)=-\frac{1}{2}, F(1, \tau)=\frac{1}{2} \text { for } \tau>0 .
\end{aligned}
$$

Taking the Laplace transform of the equation (25), we get

$$
\begin{gathered}
\frac{\partial^{2} \bar{F}}{\partial \eta^{2}}-\left[s+\frac{M^{2}}{1+m^{2}}+\frac{1}{D a}+i\left(\frac{m M^{2}}{1+m^{2}}+K^{2}+2 K_{1}^{2}\right)\right] \bar{F} \\
=-\frac{M^{2}(1+i m)}{1+m^{2}} \frac{n^{*}}{s},
\end{gathered}
$$

where

$$
\bar{F}(\eta, s)=\int_{0}^{\infty} F(\eta, \tau) e^{-s \tau} d \tau \text { and } n^{*}=s \int_{0}^{1} \bar{F}(\eta, s) d \eta .
$$

The corresponding boundary conditions for $\bar{F}(\eta, s)$ are

$$
\bar{F}(0, s)=-\frac{1}{2 s} \text { and } \bar{F}(1, s)=\frac{1}{2 s},
$$

The solution of the equation (28) subject to the boundary conditions (30) is

$$
\begin{aligned}
\bar{F}(\eta, s) & =\frac{1}{2 s}\left[\frac{\sinh \sqrt{s+a} \eta}{\sinh \sqrt{s+a}}-\frac{\sinh \sqrt{s+a}(1-\eta)}{\sinh \sqrt{s+a}}\right] \\
& -\frac{n^{*} M^{2}(1+i m)}{1+m^{2}} \times \frac{1}{s(s+a)} \\
& \times\left[\frac{\sinh \sqrt{s+a} \eta}{\sinh \sqrt{s+a}}+\frac{\sinh \sqrt{s+a}(1-\eta)}{\sinh \sqrt{s+a}}-1\right],
\end{aligned}
$$

where

$$
a=\frac{M^{2}}{1+m^{2}}+\frac{1}{D a}+i\left(\frac{m M^{2}}{1+m^{2}}+K^{2}+2 K_{1}^{2}\right) .
$$

On the use of the equation (31) in the equation (29) and on the evaluation of the integral we get $n^{*}=0$ which in turn gives $P=0$ and $Q=0$. Substituting the values of $P$ and $Q$ in the equation (16), we have

$$
E_{r}=-\omega B_{0} r \text { and } E_{\theta}=0,
$$

Substituting $n^{*}=0$, the equation (31) becomes

$$
\bar{F}(\eta, s)=\frac{1}{2 s}\left[\frac{\sinh \sqrt{s+a} \eta}{\sinh \sqrt{s+a}}-\frac{\sinh \sqrt{s+a}(1-\eta)}{\sinh \sqrt{s+a}}\right],
$$

where $a$ is given by (32).

The inverse Laplace transform of the equation (34) is

$$
\begin{aligned}
F(\eta, \tau) & =\frac{1}{2}\left[\frac{\sinh (\alpha+i \beta) \eta}{\sinh (\alpha+i \beta)}-\frac{\sinh (\alpha+i \beta)(1-\eta)}{\sinh (\alpha+i \beta)}\right] \\
& +\sum_{n=0}^{\infty} \frac{n \pi(-1)^{n}}{n^{2} \pi^{2}+a}[\sin n \pi \eta-\sin n \pi(1-\eta)] \\
& \times e^{-\left\{n^{2} \pi^{2}+(\alpha+i \beta)^{2}\right\} \tau},
\end{aligned}
$$

where

$$
\begin{aligned}
\alpha, \beta= & \frac{1}{\sqrt{2}}\left[\left\{\left(\frac{M^{2}}{1+m^{2}}+\frac{1}{D a}\right)^{2}+\left(K^{2}+2 K_{1}^{2}+\frac{m M^{2}}{1+m^{2}}\right)^{2}\right\}^{\frac{1}{2}}\right. \\
& \left. \pm\left(\frac{M^{2}}{1+m^{2}}+\frac{1}{D a}\right)\right]^{\frac{1}{2}}
\end{aligned}
$$

On separating into a real and imaginary parts one can easily obtain the velocity components $f$ and $g$ from the equation (35). If $m=0, D a=\infty$ (i.e clear fluid) and $K_{1}^{2}=0$, then the equation (35) coincides with the equation (26) of Guria et al. [8]

As $\tau \rightarrow \infty$, we get the steady state solution as

$$
F(\eta)=\frac{1}{2}\left[\frac{\sinh (\alpha+i \beta) \eta}{\sinh (\alpha+i \beta)}-\frac{\sinh (\alpha+i \beta)(1-\eta)}{\sinh (\alpha+i \beta)}\right] .
$$

If $m=0, D a=\infty$ and $K_{1}^{2}=0$ then the equation (37) reduces to the equation (22) of Mohanty [7] after simplification of the 
same.

\section{RESULTS AND DISCUSSION}

We have presented the non-dimensional primary and secondary velocities $f$ and $g$ against $\eta$ for several values of squaredHartmann number $M^{2}$, Hall parameter $m$, rotation parameters $K^{2}, K_{1}^{2}$, Darcy number $D a$ and time $\tau$ in Figs.2-7. It is observed from Fig. 2 that the velocity $f$ increases in the range $0 \leq \eta \leq 0.5$ while the reversed result occurs in the range $0.5<\eta \leq 1$ with an increase in squared-Hartmann number $M^{2}$. On the other hand, the velocity $g$ decreases in the range $0 \leq \eta \leq 0.5$ while the reversed result occurs in the range $0.5<\eta \leq 1$ with an increase in squared-Hartmann number $M^{2}$. Fig. 3 shows that the velocity $f$ decreases in the range $0 \leq \eta \leq 0.5$ while the reversed result occurs in the range $0.5<\eta \leq 1$ with an increase in Hall parameter $m$. On the other hand, the velocity $g$ increases in the range $0 \leq \eta \leq 0.5$ while the reverse result occurs in the range $0.5<\eta \leq 1$ with an increase in Hall parameter $m$. It is seen from Figs. 4 and 5 that both the velocities $f$ and $g$ increase in the range $0 \leq \eta \leq 0.5$ whereas the reversed results occur in the range $0.5<\eta \leq 1$ with an increase in either rotation parameter $K^{2}$ or $K_{1}^{2}$. This means that both the rotations have a tendency to enhance the velocity components near the lower disk whereas reduce them near the upper disk. It is observed from Figs. 6 and 7 that the velocity $f$ decreases in the range $0 \leq \eta \leq 0.5$ while the reversed result occurs in the range $0.5<\eta \leq 1$ with an increase in either Darcy number $D a$ or time $\tau$. On the other hand, the velocity $g$ increases in the range $0 \leq \eta \leq 0.5$ while the reverse result occurs in the range $0.5<\eta \leq 1$ with an increase in either Darcy number $D a$ or time $\tau$. Darcy number is the measurement of the porosity of the medium. As the porosity of the medium increases, the value of $D a$ increases. For large porosity of the medium fluid gets more space to flow as a consequence its velocity increases. It is noted that there is a symmetric motion about the mid plane between the two disks with the transparency of the rigid body rotations, which is shown by Figs. 2-6.

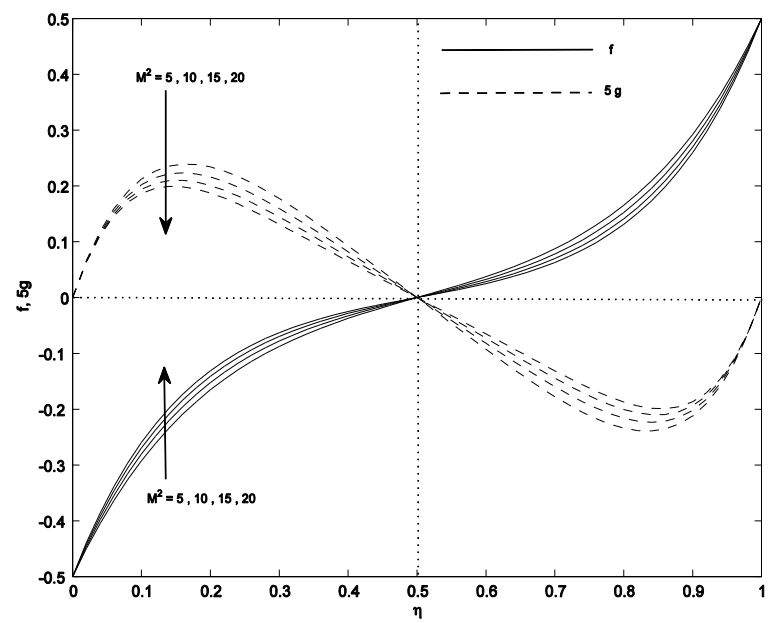

Fig.2: Primary and secondary velocities for $M^{2}$ when $m=0.2, \tau=0.2, K^{2}=5, K_{1}^{2}=5$ and $D a=0.05$

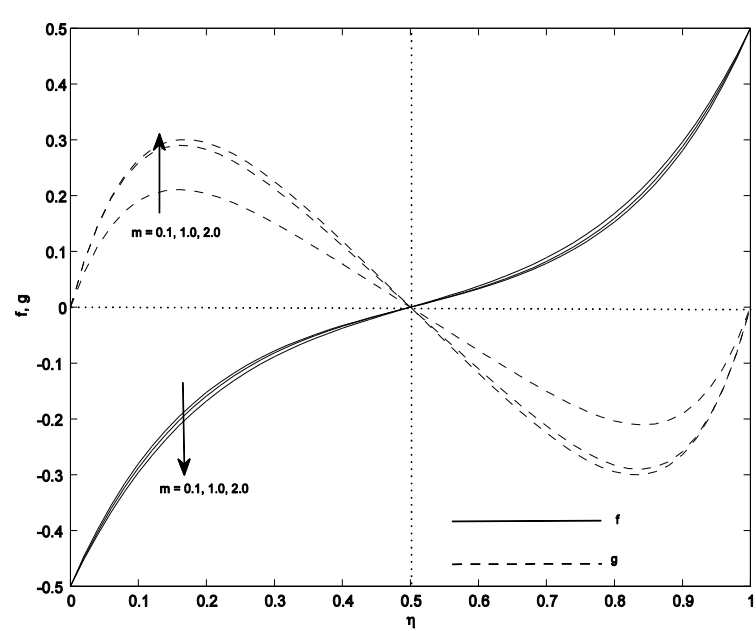

Fig.3: Primary and secondary velocities for $m$ when $M^{2}=5, \tau=0.2, K^{2}=5, K_{1}^{2}=5$ and $D a=0.05$

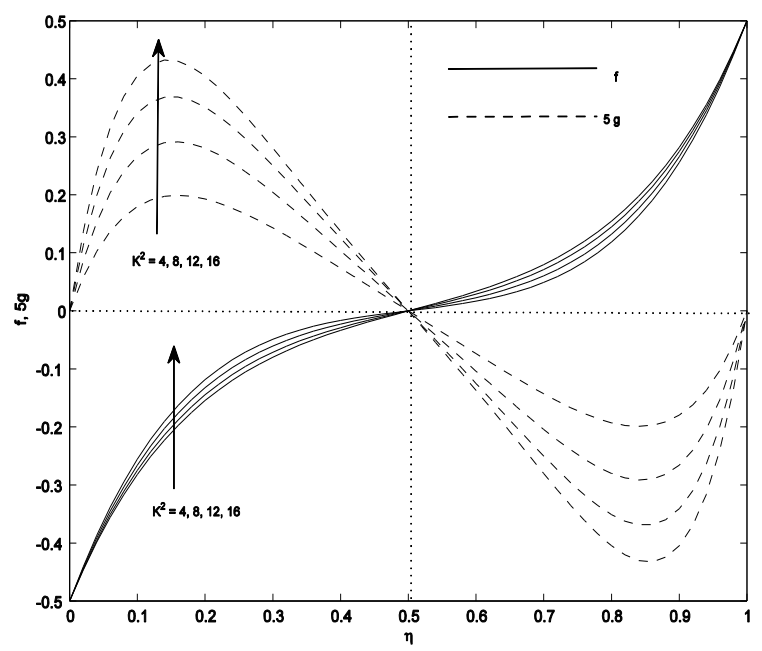

Fig.4: Primary and secondary velocities for $K^{2}$ when $M^{2}=5, \tau=0.2, m=0.2, K_{1}^{2}=5$ and $D a=0.05$

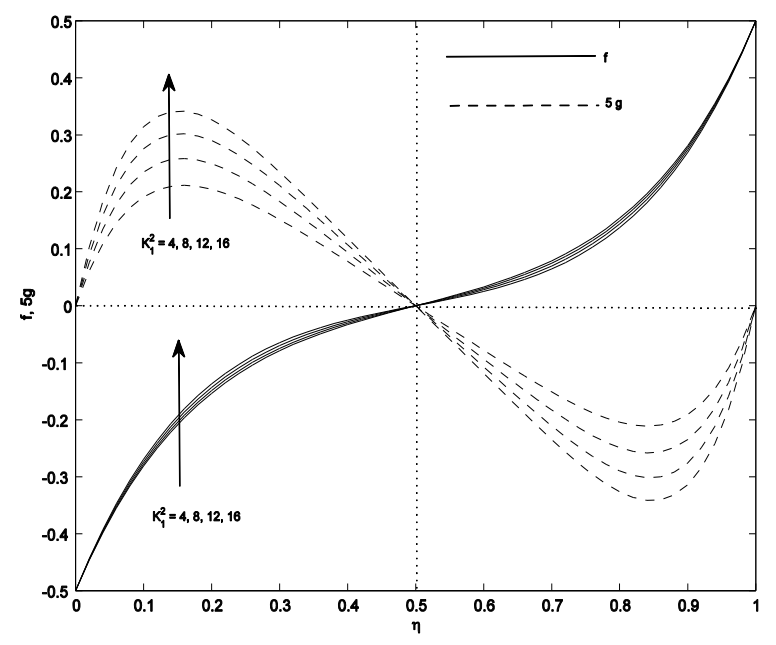

Fig.5: Primary and secondary velocities for $K_{1}^{2}$ when $M^{2}=5, \tau=0.2, m=0.2, K^{2}=5$ and $D a=0.05$ 


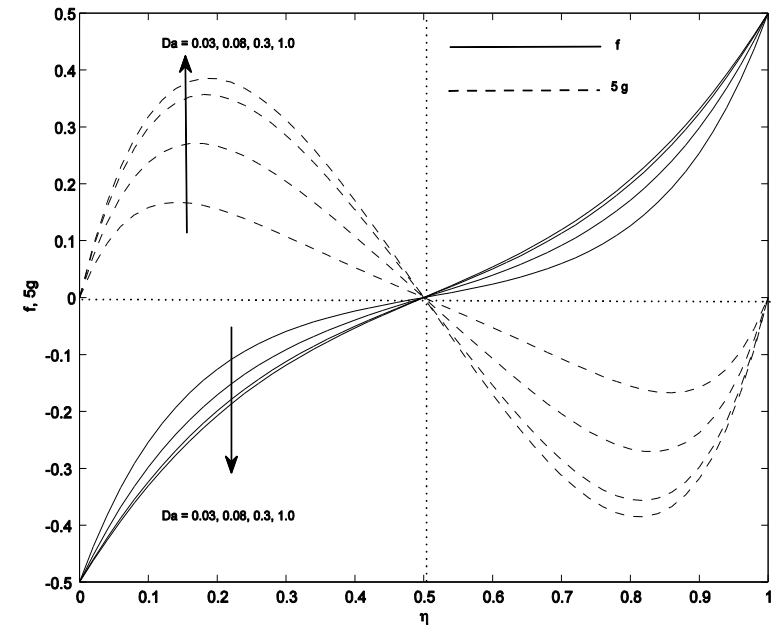

Fig.6: Primary and secondary velocities for $D a$ when $M^{2}=5, \tau=0.2, m=0.2, K_{1}^{2}=5$ and $K^{2}=5$

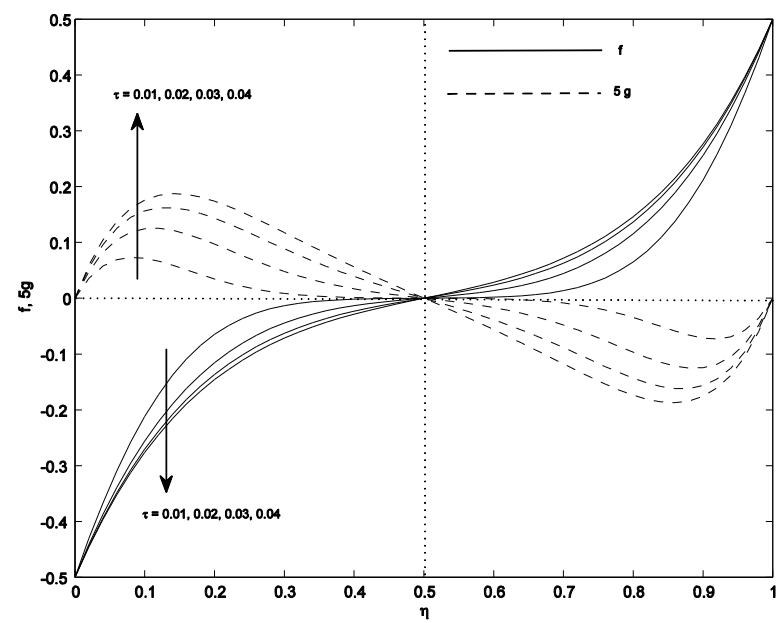

Fig.7: Primary and secondary velocities for time $\tau$ when $M^{2}=5, D a=0.05, m=0.2, K_{1}^{2}=5$ and $K^{2}=5$

The non-dimensional shear stresses due to the primary and secondary flows at the disks $\eta=0$ and $\eta=1$ are given by $\left[f^{\prime}(\eta, \tau)\right]_{(0,1)}$

$$
\begin{aligned}
& =\left(\frac{\cosh \alpha+\cos \beta}{\cosh 2 \alpha-\cos 2 \beta}\right)(\alpha \sinh \alpha+\beta \sin \beta) \\
& +\sum_{n=0}^{\infty} \frac{\pi^{2} n^{2}\left[(-1)^{n}+1\right]}{\left(\pi^{2} n^{2}+\frac{M^{2}}{1+m^{2}}+\frac{1}{D a}\right)^{2}+\left(K^{2}+2 K_{1}^{2}+\frac{m M^{2}}{1+m^{2}}\right)^{2}} \\
& \times\left[\left(\pi^{2} n^{2}+\frac{M^{2}}{1+m^{2}}+\frac{1}{D a}\right) \cos \left(K^{2}+2 K_{1}^{2}+\frac{m M^{2}}{1+m^{2}}\right) \tau\right. \\
& \left.-\left(K^{2}+2 K_{1}^{2}+\frac{m M^{2}}{1+m^{2}}\right) \sin \left(K^{2}+2 K_{1}^{2}+\frac{m M^{2}}{1+m^{2}}\right) \tau\right] \\
& \times e^{-\left(\frac{M^{2}}{1+m^{2}}+\frac{1}{D a}+\pi^{2} n^{2}\right) \tau},
\end{aligned}
$$

$\left[g^{\prime}(\eta, \tau)\right]_{(0,1)}$

$$
\begin{aligned}
& =\left(\frac{\cosh \alpha+\cos \beta}{\cosh 2 \alpha-\cos 2 \beta}\right)(\beta \sinh \alpha-\alpha \sin \beta) \\
& -\sum_{n=0}^{\infty} \frac{\pi^{2} n^{2}\left[(-1)^{n}+1\right]}{\left(\pi^{2} n^{2}+\frac{M^{2}}{1+m^{2}}+\frac{1}{D a}\right)^{2}+\left(K^{2}+2 K_{1}^{2}+\frac{m M^{2}}{1+m^{2}}\right)^{2}} \\
& \times\left[\left(\pi^{2} n^{2}+\frac{M^{2}}{1+m^{2}}+\frac{1}{D a}\right) \sin \left(K^{2}+2 K_{1}^{2}+\frac{m M^{2}}{1+m^{2}}\right) \tau\right. \\
& \left.+\left(K^{2}+2 K_{1}^{2}+\frac{m M^{2}}{1+m^{2}}\right) \cos \left(K^{2}+2 K_{1}^{2}+\frac{m M^{2}}{1+m^{2}}\right) \tau\right] \\
& \times e^{-\left(\frac{M^{2}}{1+m^{2}}+\frac{1}{D a}+\pi^{2} n^{2}\right) \tau} .
\end{aligned}
$$

The torque required to overcome the transverse shearing stress on a disk of radius $b$ is

$$
\begin{aligned}
& \tau_{1}=2 \int_{0}^{b} \int_{0}^{\pi} \tau_{z \theta} r^{2} d r d \theta \\
& =-\frac{1}{3} \frac{\rho v \omega a b^{3}}{h}\left[\left(\frac{\cosh \alpha+\cos \beta}{\cosh 2 \alpha-\cos 2 \beta}\right)(\alpha \sinh \alpha+\beta \sin \beta)\right. \\
& +\sum_{n=0}^{\infty} \frac{\pi^{2} n^{2}\left[(-1)^{n}+1\right]}{\left(\pi^{2} n^{2}+\frac{M^{2}}{1+m^{2}}+\frac{1}{D a}\right)^{2}+\left(K^{2}+2 K_{1}^{2}+\frac{m M^{2}}{1+m^{2}}\right)^{2}} \\
& \times\left[\left(\pi^{2} n^{2}+\frac{M^{2}}{1+m^{2}}+\frac{1}{D a}\right) \cos \left(K^{2}+2 K_{1}^{2}+\frac{m M^{2}}{1+m^{2}}\right) \tau\right. \\
& \left.-\left(K^{2}+2 K_{1}^{2}+\frac{m M^{2}}{1+m^{2}}\right) \sin \left(K^{2}+2 K_{1}^{2}+\frac{m M^{2}}{1+m^{2}}\right) \tau\right] \\
& \times e^{-\left(\frac{M^{2}}{1+m^{2}}+\frac{1}{D a}+\pi^{2} n^{2}\right) \tau} \\
& \tau_{0}=\frac{\tau_{1}}{-\frac{1}{3} \frac{\rho v \omega a b^{3}}{h}}=\left(\frac{\cosh \alpha+\cos \beta}{\cosh 2 \alpha-\cos 2 \beta}\right)(\alpha \sinh \alpha+\beta \sin \beta) \\
& +\sum_{n=0}^{\infty} \frac{\pi^{2} n^{2}\left[(-1)^{n}+1\right]}{\left(\pi^{2} n^{2}+\frac{M^{2}}{1+m^{2}}+\frac{1}{D a}\right)^{2}+\left(K^{2}+2 K_{1}^{2}+\frac{m M^{2}}{1+m^{2}}\right)^{2}} \\
& \times\left[\left(\pi^{2} n^{2}+\frac{M^{2}}{1+m^{2}}+\frac{1}{D a}\right) \cos \left(K^{2}+2 K_{1}^{2}+\frac{m M^{2}}{1+m^{2}}\right) \tau\right. \\
& \left.-\left(K^{2}+2 K_{1}^{2}+\frac{m M^{2}}{1+m^{2}}\right) \sin \left(K^{2}+2 K_{1}^{2}+\frac{m M^{2}}{1+m^{2}}\right) \tau\right] \\
& \times e^{-\left(\frac{M^{2}}{1+m^{2}}+\frac{1}{D a}+\pi^{2} n^{2}\right) \tau} .
\end{aligned}
$$

The numerical values of the torque $\tau_{0}$ at the disks are presented in Figs.8-12 for several values of squared-Hartmann number $M^{2}$, rotation parameters $K^{2}, K_{1}^{2}$, Darcy number $D a$ and time $\tau$ against Hall parameter $m$. It is seen from Figs.8-10 that the torque $\tau_{0}$ at the disks increases with an increase in either $M^{2}$ or $K^{2}$ or $K_{1}^{2}$. Figs.11 and 12 show that the torque $\tau_{0}$ at the disks decreases with an increase in either $D a$ or $\tau$. Further, it is observed from Figs.8-12 that the torque $\tau_{0}$ at the disks decreases with an increase in Hall parameter $m$. 


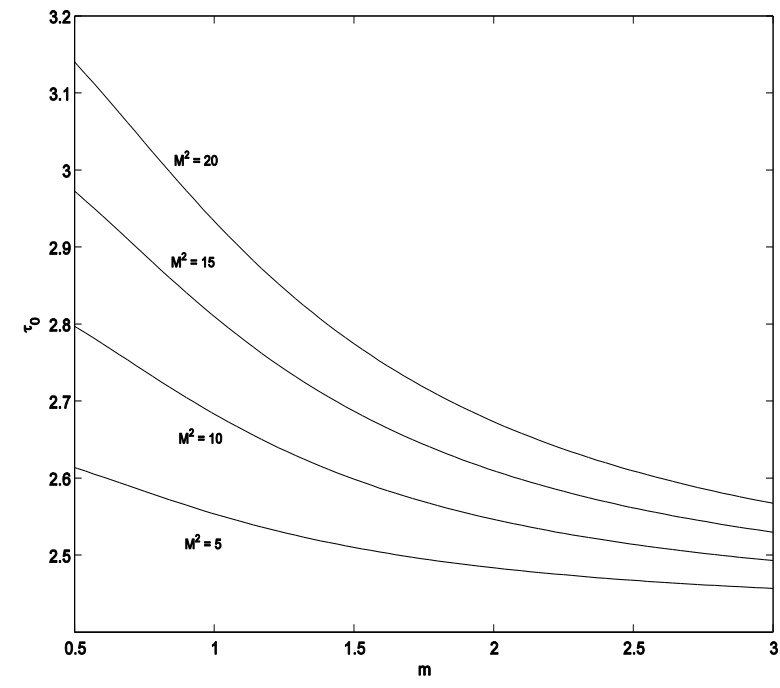

Fig.8: Variation of torque $\tau_{0}$ for $M^{2}$ when $\tau=0.05, K^{2}=5$ , $K_{1}^{2}=5$ and $D a=0.05$

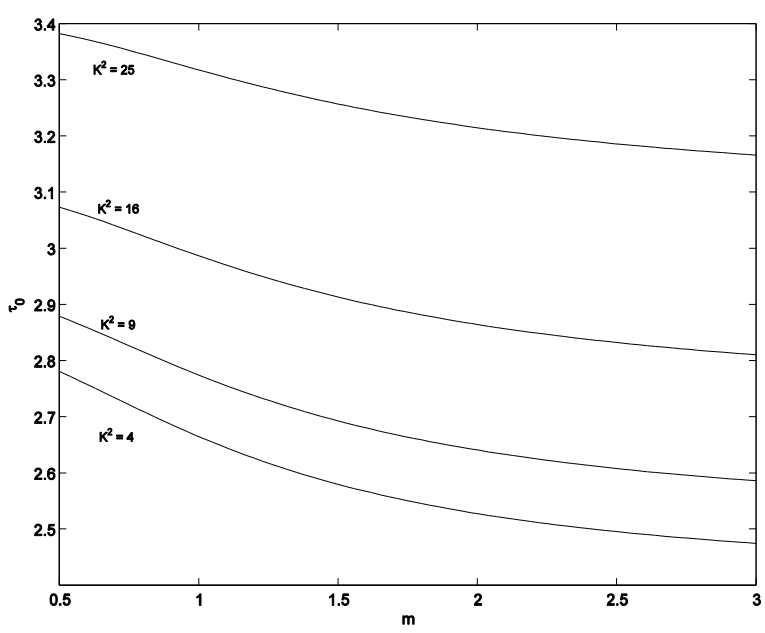

Fig.9: Variation of torque $\tau_{0}$ for $K^{2}$ when $M^{2}=5$, $\tau=0.05, K_{1}^{2}=5$ and $D a=0.05$

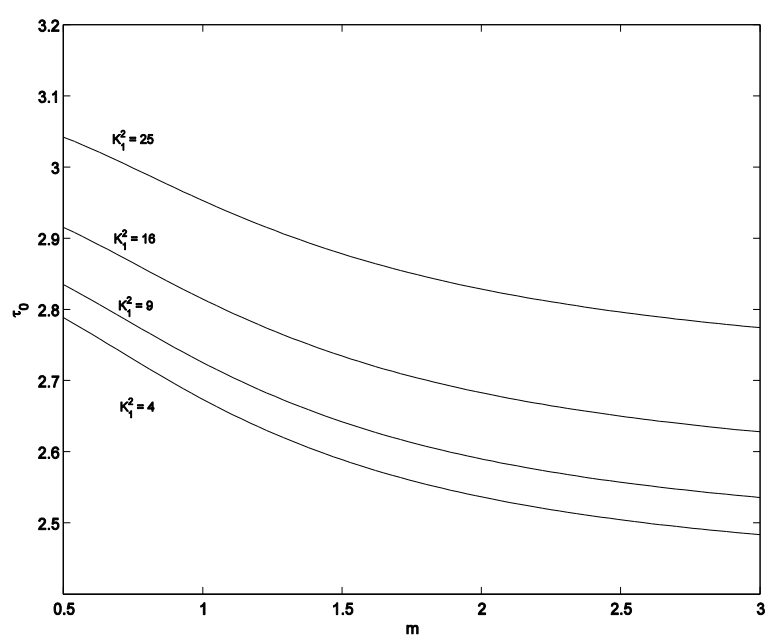

Fig.10: Variation of torque $\tau_{0}$ for $K_{1}^{2}$ when $M^{2}=5$, $\tau=0.05, K^{2}=5$ and $D a=0.05$

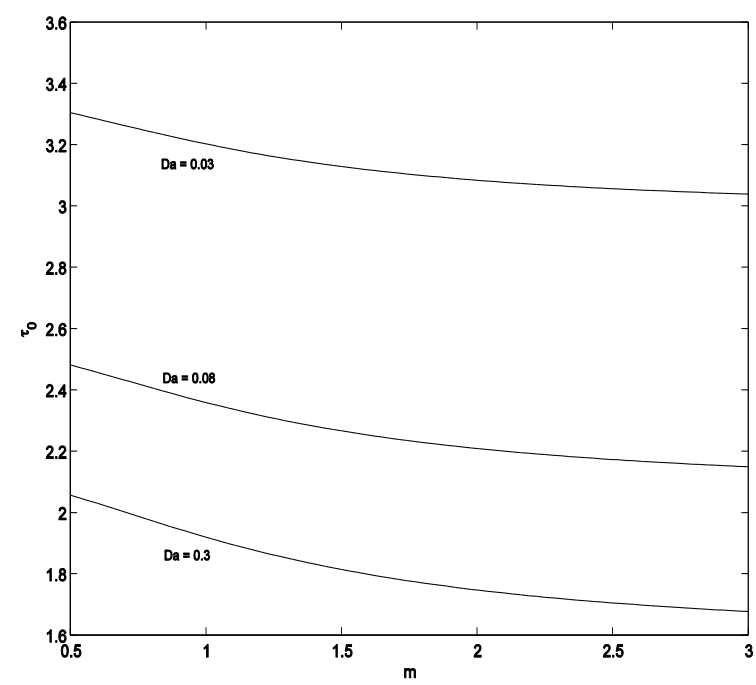

Fig.11: Variation of torque $\tau_{0}$ for $D a$ when $M^{2}=5$, $\tau=0.05, K^{2}=5$ and $K_{1}^{2}=5$

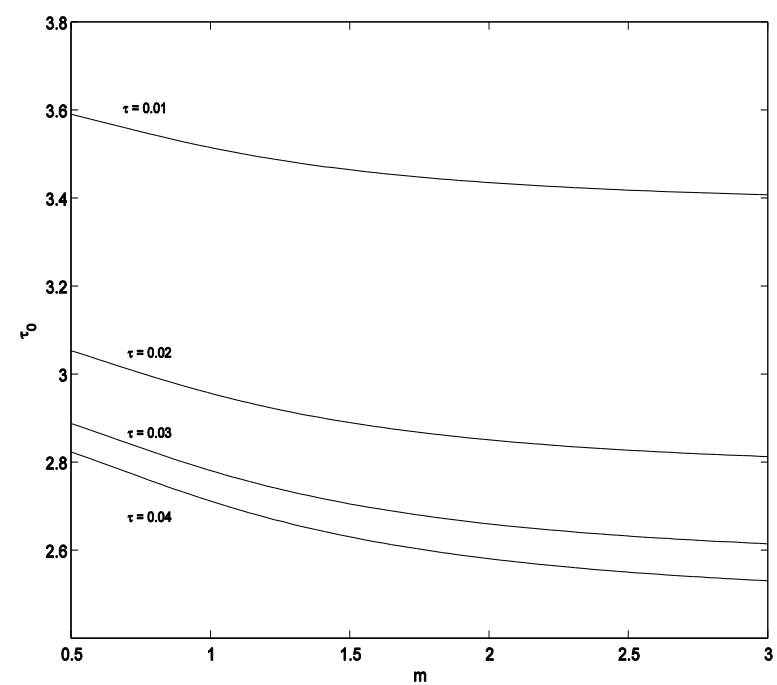

Fig.12: Variation of torque $\tau_{0}$ for time $\tau$ when $M^{2}=5$,

$$
K^{2}=5, K_{1}^{2}=5 \text { and } D a=0.05
$$

It is seen from (40) that in the steady state ( i.e. $\tau \rightarrow \infty$ ), the torque $\tau_{1}$ becomes

$$
\tau_{1}=-\frac{1}{3} \frac{\rho \nu \Omega a b^{3}}{h}\left[\left(\frac{\cosh \alpha+\cos \beta}{\cosh 2 \alpha-\cos 2 \beta}\right)(\alpha \sinh \alpha+\beta \sin \beta)\right],
$$

This steady state is reached through the inertial oscillations with frequency

$$
\omega=K^{2}+2 K_{1}^{2}+\frac{m M^{2}}{1+m^{2}} .
$$

The equation (42) coincides with the equation (24) of Mohanty [7] when $m=0, D a=\infty$ and $K_{1}^{2}=0$.

\section{CONCLUSION}

Hall effects on an unsteady MHD flow through a porous medium between two disks rotating with the same uniform angular velocity about two different axes have been studied. It is observed that both the rotation parameters have a tendency to enhance the velocity components near the lower disk whereas reduce them near the upper disk. The torque required to overcome the transverse shearing stress on the disks increases 
with an increase in either squared-Hartmann number or rotation parameters while it decreases with an increase in either Hall parameter or Darcy number. The significance of the study of this problem of MHD rotating flows is that there exists symmetric solution of this problem with regard to a rigid body rotation.

\section{REFERENCES}

[1] Stewatson, K. (1953). On the flow between two rotating co-axial disks, Proc. Cambridge Phi. Soc., 49: 533-541.

[2] Rott, N. and Lewellen, W. S. (1966). Boundary layer and their interactions in rotating flows, Prog. aeronaut. Sci. ,7:111-144.

[3] Mellor, G.L., Chapple, P. J. and Stocks, V. K. (1968). On the flow betweena rotating and a stationary disk, J. Fluid Mech., 31: 95-112.

[4] Erdogan, M. E. (1995). Unsteady viscous flow between eccentric disks, Int. J. Non-linear Mech., 30: 711-717.

[5] Ersoy, H. V.(2003). Unsteady flow due to concentric rotation of ecentric rotating disks, Meceanica, 38: 325-334.

[6] Jana, R. N., Jana, M. Das, S., Maji, S. L. and Ghosh, S. K. (2011). Hydrodynamic flow between two non-coincident rotating disks embedded in porous media, World $J$. Mechanics, 1: 50-56.

[7] Mohanty, H. K. (1971). Hydromagnetic flow between two rotating disks with non-coincident parallel axes of rotation, Phys. Fluids, 15: 1456-1458.

[8] Guria, M., Das, S. and Jana, R. N.(2007). Hall effects on unsteady flow of a viscous fluid due to non-coaxial rotation of a porous disk and a fluid at infinity, Int. J. Nonlinear Mech., 30:1204-1209.

[9] Guria, M., Das, B. K., Jana, R. N. and Imrak, C. E.(2008). Hydromagnetic flow between two porous disks rotating about non-coincident axes, Acta Mechanica Sinica, 24(5): 489-496.

[10] Sutton, G. and Sherman, A. (1965). Engineering Magnetohydrodynamics, McGraw-Hill, New York.
[11] Tillack, M. S. and Morley, N. B. (1998). Standard Handbook for Electrical Engineers: Magnetodynamics, McGraw-Hill, New York.

[12] Sato, H. (1961). The Hall effects in the viscous flow of ionized gas between parallel plates under transverse magnetic field, J. Phys. Soc. Jpn., 16: 1427.

[13] Yamanishi,T. (1962). Preprint, 17th Annual meeting, Phys.Soc. Jpn., Osaka.

[14] Sherman, A. and Sutton, G. W.(1965). Engineering Magnetohydrodynamics, McGraw-Hill,Inc, New York.

[15] Gupta, A. S. (1975). Hydromagnetic flow past a porous flat plate with Hall effects, Acta Mechanica, 22: 281-267.

[16] Datta, N.and Jana,R. N. (1976). Oscillatory magnetohydrodynanic flow past a flat plate with Hall effects, J. Phys. Soc. Jpn., 40: 1469-1474.

[17] Datta, N. and Jana, R. N. (1977). Hall effects on hydromagnetic convective flow through a channel with conducting walls, Int. J. Engng Sci., 15: 561.

[18] Jana, R. N. and Datta, N.(1977). Hall effects on unsteady Couette flow, Int. J. Engng Sci., 15: 35.

[19] Kanch, A. K. and Jana, R. N. (1992). Hall effects on hydromagnetic flow between two rotating disks with noncoincident parallel axes of rotation, Rev. Roumsci. Tech. Mech. Appl., 37: 379-385.

[20] Das, S., Maji, S. L., Guria, M. and Jana, R. N.(2010). Hall effects on unsteady MHD flow between two disks with non-coincident parallel axes of rotation, Int. J. Appl. Mech. Eng., 15(1): 5-18.

[21] Barik, R. N., Das, G. C. and Rath, P. K.(2013). Hall effects on unsteady MHD flow between two rotating disks with non-coincident parallel axes, Proc. Natl. Acad. Sci. India Sect. A Phys. Sci., 83(1) : 21-27.

[22] Cowling, T.G. (1957). Magnetohydrodynamics, Interscience Publisher, Inc, New York. 